IOS Press

\title{
Is Losing One's Way a Sign of Cognitive Decay? Topographical Memory Deficit as an Early Marker of Pathological Aging
}

\author{
Maddalena Boccia ${ }^{\mathrm{a}, 1, *}$, Antonella Di Vita ${ }^{\mathrm{a}, \mathrm{b}, \mathrm{c}, 1}$, Sofia Diana ${ }^{\mathrm{b}, \mathrm{c}}$, Roberta Margiotta ${ }^{\mathrm{b}, \mathrm{c}}$, \\ Letizia Imbriano $^{\mathrm{c}}$, Lidia Rendace $^{\mathrm{c}}$, Alessandra Campanelli ${ }^{\mathrm{c}}$, Fabrizia D’Antonio ${ }^{\mathrm{c}}$, \\ Alessandro Trebbastoni ${ }^{\mathrm{c}}$, Carlo de Lena ${ }^{\mathrm{c}}$, Laura Piccardi ${ }^{\mathrm{a}, \mathrm{d}}$ and Cecilia Guariglia ${ }^{\mathrm{a}, \mathrm{b}}$ \\ ${ }^{a}$ Cognitive and Motor Rehabilitation Unit, IRCCS Fondazione Santa Lucia, Rome, Italy \\ ${ }^{\mathrm{b}}$ Department of Psychology, Sapienza University of Rome, Rome, Italy \\ 'Department of Human Neuroscience, "Sapienza" University of Rome, Rome, Italy \\ ${ }^{\mathrm{d}}$ Department of Life, Health and Environmental Sciences, L'Aquila University, L'Aquila, Italy
}

Accepted 14 January 2019

\begin{abstract}
Spatial navigation tasks reveal small differences between normal and pathological aging and may thus disclose potential neuropsychological predictors of neurodegenerative diseases. The aim of our study was to investigate which navigational skills are compromised in the early phase of pathological aging as well as the extent to which they are compromised. We performed an extensive neuropsychological evaluation based on working memory and learning tasks (i.e., Corsi BlockTapping Test and Walking Corsi Test) involving both reaching and navigational vista spaces. We also assessed spatial navigation skills in the real world by asking participants to perform route-learning and landmark-recognition tasks. We conducted a cross-sectional study on nineteen patients with a diagnosis of mild cognitive impairment (MCI) who displayed either an isolated memory deficit (single-domain amnestic MCI, MCIsd; $\mathrm{N}=3$ ) or a memory deficit associated with deficits in other cognitive functions (multi-domain MCI, MCImd; $\mathrm{N}=16$ ) as well as on nineteen healthy control participants. The groups' performances were compared by means of mixed factorial ANOVA and two-sample $t$-tests. We found that patients with MCI performed worse than controls, especially when they were required to learn spatial positions within the navigational vista space. Route-learning within the real environment was also impaired whereas landmark-recognition was spared. The same pattern of results emerged in the MCImd subgroup. Moreover, single case analyses on MCIsd patients revealed a dissociation between learning of spatial positions within navigational vista space and within reaching space. These results suggest that topographical learning is compromised in the early phase of MCIsd and MCImd and that spatial navigation tasks may be used to better characterize topographical disorientation in MCI patients as well as for the early diagnosis of pathological aging.
\end{abstract}

Keywords: Alzheimer's disease, environmental navigation, mild cognitive impairment, topographical memory

\section{INTRODUCTION}

In the last 20 years, the increase in life expectancy and prevalence of age-related cognitive disorders has led to a growing interest in predictors of

\footnotetext{
${ }^{1}$ These authors contributed equally to this work.

*Correspondence to: Maddalena Boccia, $\mathrm{PhD}$, Cognitive and Motor Rehabilitation Unit, IRCCS Fondazione Santa Lucia, via Ardeatina 306, 00179, Rome, Italy. Tel.: +390651501118; E-mail: m.boccia@hsantalucia.it.
}

neurodegenerative diseases. The identification of markers of pathological aging that may lead to an early diagnosis and the prompt initiation of cognitive stimulation treatments or pharmacological treatments is, however, a challenging task. In this regard, the study of ability of humans to orient themselves in the environment, i.e., environmental navigation, is a particularly interesting issue since getting lost is often the very first symptom of pathological aging and of some neurodegenerative diseases, such as 
Alzheimer's disease (AD) and mild cognitive impairment [1]. As occurs in other cognitive functions, environmental navigation declines both in normal aging [2-4] and in some neurodegenerative diseases associated with aging, such as mild cognitive impairment and AD [5-7]. Any differences between normal and pathological aging in how environmental navigation declines may disclose neuropsychological predictors of neurodegenerative diseases.

However, spatial navigation is a multifaceted skill [8] that results from the interaction between a number of abilities. To successfully navigate towards an environmental goal, we have to 1) correctly locate ourselves in the environment, 2) identify our goal, and 3) plan the route toward our goal. To achieve these steps, we access at least two types of spatial representations, i.e., the online representation of our position in the environment and the offline representation of the environment [9]. Moreover, whenever we navigate in environmental space, we acquire new spatial information related to the location, distance and direction, which we store and subsequently retrieve when we try to reach a familiar place or give other people directions to a spatial position [10]. It may be possible to detect neuropsychological predictors of neurodegenerative diseases by examining which components of the spatial navigation system are compromised in elderly subjects.

Some recent studies aimed at investigating which components of the spatial navigation system are compromised in elderly people identified distinct aspects of this complex skill. The ability to create configurational representations of the environment appears to be particularly compromised in healthy elderly subjects. Indeed, elderly people present a general decline in the ability to form and use cognitive maps of new environments as they display a difficulty in extracting information from the environment to effectively orient themselves, to remember a pre-established path and to recognize salient places [2,3]. Older people also less accurately recognize images (or videos) of places along a previously learned path $[4,7$, 11], a weakness that persists following an extensive learning procedure [12]. Deficits in environmental navigation in normal and pathological aging are qualitatively similar (deficits in the creation of a cognitive map of the environment and egocentric deficits) and can only be differentiated at the quantitative level [7]. Recent experimental evidence has not only confirmed these findings by means of paradigms designed to study learning and recalling information in different formats of representing space (i.e., the allocentric and/or egocentric frameworks), but has demonstrated a concomitant neuro-functional alteration in regions classically involved in navigation [13]. Taken as a whole, these data point to the existence of a continuum between the impairment in navigation skills in healthy elderly subjects [12] and that in those affected by pathological aging [13]. Is there a critical boundary between these two groups that may be used as a neuropsychological marker of pathological aging? Studying navigation skills within the transitional zone between normal and pathological aging may help to answer this question.

Mild cognitive impairment has been described as a "transitional zone" between normal and pathological aging. This term is used to refer to elderly individuals with a very mild degree of cognitive decline. The identification of early predictors of dementia in this pre-clinical phase may be crucial, and environmental navigation may be one such predictor. Indeed, several studies have reported that disorders in spatial orientation (topographical disorientation, TD) are considered an early symptom of dementia [14-18). The prevalence of TD in the later stages of $\mathrm{AD}$ is reported to range from $40 \%$ to $54 \%[19,20]$. Studies in the literature point to the presence in patients diagnosed with $\mathrm{AD}$ and mild cognitive impairment of deficits in their ability to create a map of the environment, to process the egocentric representation of the pathways [21] and to retrieve stored allocentric environmental information [22, 23].

A recently published study shows that patients with amnestic mild cognitive impairment (henceforth referred to as MCI) commit more errors in spatial navigation in a real environment than controls, particularly errors in turns [24]. Such patients also display impairments in storing new topographical memories from an allocentric (map-like) perspective and in retrieving stored allocentric information to perform an egocentric task, i.e., to use a previously acquired map-like representation to determine whether the proposed direction (i.e., left, straight, right) is the one they need to follow to reach the next crossroad along the path; these deficits are related to specific patterns of brain atrophy and hypometabolism in the neural network of environmental navigation [13], which consists of the medial temporal lobe structure (including the hippocampus and the parahippocampal gyrus), the parietal lobe (including the retrosplenial complex and the inferior parietal lobule) and the frontal lobe [25]. A clear dissociation between spatial memory within reaching and navigational vista space [26] has also been 
reported in early AD patients, who performed significantly worse than healthy age-matched controls when tested on the Walking Corsi Test (WalCT) [27, 28], a larger version of the classical Corsi BlockTapping Test (CBT) [29]; by contrast, no differences emerged when the two groups were tested on the CBT [10]. This clearly points towards a possible dissociation between spatial memory within reaching space and spatial memory within navigational vista space in pathological aging as well. Thus, spatial memory within navigational vista space (hereafter called topographical memory) may be a neuropsychological marker of $\mathrm{AD}$ in MCI which should be further explored.

In the light of the continuum between normal and pathological aging described above, it is of paramount importance to identify spatial navigation tasks that can detect small differences between normal and pathological aging. In addition to topographical memory [10], two other tasks that are hypothesized to be particularly sensitive to early signs of $\mathrm{AD}$ [30] and to the development of dementias are wayfinding and route learning [31, 32].

The aim of our study was to investigate which navigational skills are compromised in patients with MCI, compared with healthy controls, as well as the extent to which they are compromised. For this purpose, we evaluated working memory and spatial learning in both reaching and navigational vista spaces, which are spaces that can be visually inspected from a single location or by means of small exploratory movements. We also tested spatial navigation skills in the real world environmental space, which is instead space that cannot be inspected from a single location but requires a considerable number of movements within the environment [26].

\section{MATERIALS AND METHODS}

\section{Sample description}

Nineteen patients with a diagnosis of MCI (9 women; mean age $=74.89, \mathrm{SD}=5.37$; mean education $=10.32, \mathrm{SD}=4.75)$ and 19 healthy controls (hereinafter called C) ( 13 women; mean age $=73.74$, $\mathrm{DS}=5.39$; mean education $=10.63, \mathrm{SD}=4.36)$ took part in this study. The two groups were matched for Age $\left(\mathrm{t}_{36}=-0.66 ; p=0.512\right)$, Gender $\left(\chi^{2}=1.73\right.$; $p=0.19)$, and Education $\left(\mathrm{t}_{36}=0.21 ; p=0.832\right)$. No participants had a history of psychiatric disease, alcohol or drug abuse, traumatic brain injury, acute cerebrovascular disorders, or severe central nervous system infections.
Patients with MCI were recruited at the Cognitive Disorders and Dementia Center (CDCD), "Policlinico Umberto I" University Hospital of Rome. The diagnosis of MCI was made by expert neurologists according to clinical criteria from the National Institute on Aging-Alzheimer's Association workgroups (NIA-AA) [33]. All the patients had a clinical history of memory deficits, though their functional abilities and autonomy in daily life were fully preserved. Three MCI patients had an isolated memory deficit (hereinafter called single-domain amnestic MCI, MCIsd) and 16 had a memory deficit associated with other cognitive function deficits (hereinafter called multi-domain MCI, MCImd). All the MCI patients had a Clinical Dementia Rating score (CDR) of 0.5 and did not display any motor deficits that might have hampered the execution of the tests. Patients were excluded if they had secondary causes of cognitive decline (i.e., vitamin deficiency or severe hypothyroidism, hydrocephalus, syphilis, alcohol abuse), psychiatric comorbidities or a history of head trauma, had had severe central nervous system infections within the last 5 years, or had a history of cerebrovascular disease (i.e., stroke, transient ischemic attacks, cerebral hemorrhage). The demographic characteristics and clinical data are shown in Table 1.

None of the $\mathrm{C}$ had a history of neurologic or psychiatric diseases. The Mini-Mental State Examination (MMSE $\geq 27$ ) [34] and the Alzheimer's Disease Assessment Scale-Cognitive subscale (ADAS-Cog $11 \leq 15$ ) scores of all the $\mathrm{C}$ fell within the normal range [35].

The study was designed in accordance with the ethical principles of Human experimentation stated in the Declaration of Helsinki and was approved by the Local Ethics Committee of "Policlinico Umberto I" in Rome. All the participants provided written informed consent after the procedures had been fully explained to them.

\section{Procedure}

The neuropsychological and experimental tasks described below were administered on two separate days. All the participants were initially asked to provide anamnestic information (e.g., previous illness, neurological and/or psychiatric history, medication). On the first day, they underwent a general and neurological clinical evaluation as well as neuropsychological testing including the assessment of: 1) memory skills, by using the Rey Auditory Verbal 
Table 1

Control and MCI demographic characteristics

\begin{tabular}{|c|c|c|c|c|c|c|c|c|}
\hline Sub-group & Subject & Gender & Age & Education & $A D L$ & $I A D L$ & $M M S E$ & $A D A S-C o g$ \\
\hline \multirow[t]{16}{*}{ MCImd } & MCImd1 & $F$ & 75 & 5 & 6 & 8 & 24 & 25 \\
\hline & MCImd2 & $M$ & 73 & 8 & 6 & 5 & 23 & 17 \\
\hline & MCImd3 & $F$ & 71 & 17 & 6 & 8 & 25 & 13 \\
\hline & MCImd4 & $F$ & 63 & 8 & 6 & 8 & 26 & 19 \\
\hline & MCImd5 & $M$ & 75 & 8 & 6 & 5 & 25 & 15 \\
\hline & MCImd6 & $F$ & 80 & 5 & 6 & 8 & 22 & 19 \\
\hline & MCImd7 & $F$ & 65 & 13 & 6 & 8 & 26 & 35 \\
\hline & MCImd8 & $F$ & 81 & 8 & 6 & 8 & 24 & 18 \\
\hline & MCImd9 & $M$ & 74 & 5 & 6 & 5 & 24 & 21 \\
\hline & MCImd10 & $F$ & 82 & 5 & 6 & 8 & 21 & 16 \\
\hline & MCImd11 & $M$ & 77 & 13 & 4 & 5 & 25 & 24 \\
\hline & MCImd12 & $M$ & 76 & 8 & 6 & 5 & 22 & 27 \\
\hline & MCImd13 & $M$ & 79 & 8 & 6 & 5 & 26 & 22 \\
\hline & MCImd14 & $M$ & 81 & 17 & 6 & 5 & 26 & 24 \\
\hline & MCImd15 & $M$ & 76 & 17 & 6 & 5 & 25 & 13 \\
\hline & MCImd16 & $M$ & 70 & 5 & 6 & 5 & 20 & 23 \\
\hline \multirow[t]{3}{*}{ MCIsd } & MCIsdl & $F$ & 80 & 16 & 6 & 8 & 29 & 22 \\
\hline & $M C I s d 2$ & $F$ & 76 & 13 & 6 & 8 & 28 & 25 \\
\hline & MCIsd3 & $M$ & 69 & 17 & 6 & 5 & 29 & 18 \\
\hline \multirow[t]{19}{*}{$C$} & $C 1$ & $\mathrm{~F}$ & 79 & 17 & 6 & 8 & 29 & 5 \\
\hline & $C 2$ & M & 79 & 13 & 6 & 8 & 28 & 15 \\
\hline & C3 & $\mathrm{F}$ & 73 & 8 & 6 & 8 & 28 & 7 \\
\hline & $C 4$ & M & 69 & 13 & 6 & 5 & 29 & 7 \\
\hline & $C 5$ & $\mathrm{M}$ & 77 & 5 & 6 & 5 & 30 & 7 \\
\hline & C6 & $\mathrm{F}$ & 76 & 5 & 6 & 8 & 27 & 10 \\
\hline & $C 7$ & F & 69 & 10 & 6 & 8 & 30 & 7 \\
\hline & C8 & $\mathrm{F}$ & 66 & 5 & 6 & 8 & 30 & 6 \\
\hline & $C 9$ & M & 64 & 11 & 6 & 5 & 27 & 9 \\
\hline & $C 10$ & $\mathrm{~F}$ & 69 & 5 & 6 & 8 & 30 & 7 \\
\hline & $C 11$ & $\mathrm{~F}$ & 82 & 17 & 6 & 8 & 30 & 9 \\
\hline & $C 12$ & $\mathrm{~F}$ & 68 & 13 & 6 & 8 & 30 & 8 \\
\hline & $C 13$ & M & 83 & 18 & 6 & 5 & 29 & 8 \\
\hline & C14 & F & 72 & 13 & 6 & 8 & 28 & 5 \\
\hline & $C 15$ & $\mathrm{M}$ & 78 & 10 & 6 & 5 & 30 & 7 \\
\hline & C16 & $\mathrm{F}$ & 72 & 13 & 6 & 8 & 29 & 7 \\
\hline & $C 17$ & F & 76 & 8 & 6 & 8 & 30 & 5 \\
\hline & C18 & $\mathrm{F}$ & 72 & 13 & 6 & 8 & 29 & 8 \\
\hline & C19 & F & 77 & 5 & 6 & 8 & 29 & 9 \\
\hline
\end{tabular}

MCImd, multi-domain mild cognitive impairment; MCIsd, single-domain mild cognitive impairment; C, control; MMSE, Mini-Mental State Examination; ADAS-Cog, Alzheimer's Disease Assessment Scale-cognitive subscale; RCPM, Raven's Colored Progressive Matrices; ADL, Activities of Daily Living; IADL, Instrumental Activities of Daily Living.

Learning Test [36], Rey-Osterrieth complex figure test [36], Corsi block-tapping test [37], Digit Span test [38], Babcock Story Recall Test [39]; 2) attention, by means of Visual Search [38], Trail Making Test [38]; 3) language, by means of the Phonemic Verbal Fluency task [40], Semantic Verbal Fluency task [40], Token Test [38], Boston Naming Test [41]; 4) executive functions, by means of the Frontal Assessment Battery [42]; 5) logic abstract reasoning, by means of Raven's Colored Progressive Matrices [43, 44]; and 6) visuo-constructional skills, by means of Copy of Rey-Osterrieth's Complex Figure test [36] and Clock Test [45]. Averaged MCI performances are reported in Table 2. General cognitive function was assessed by means of the MMSE and ADAS-Cog $11[34,35] . \quad 260$ Functional and instrumental activities of daily living $\quad 261$ (ADL/IADL; [46]) were also investigated in MCI; ${ }_{262}$ scores are reported in Table 1.

On the second day, participants underwent the CBT $\quad{ }_{264}$ and WalCT to assess visuo-spatial and topographical 265 memory, respectively. Environmental navigation in $\quad 266$ the real world was also investigated in the same ses- $\quad 267$ sion. These tasks will be thoroughly discussed below. $\quad 268$

Visuo-spatial memory: Reaching space

Visuo-spatial working memory (VSWM) and 270 learning (VSL) were tested in reaching space by 271 
Table 2

Patients' and controls' scores in neuropsychological tests

\begin{tabular}{|c|c|c|c|c|c|c|c|c|c|}
\hline \multirow[t]{2}{*}{ Test } & \multicolumn{2}{|c|}{$\mathrm{C}$} & \multicolumn{2}{|c|}{ MCImd } & \multicolumn{2}{|c|}{ Levene Test } & \multicolumn{3}{|c|}{ Two-sample $t$-tests } \\
\hline & $\mathrm{M}$ & SD & $\mathrm{M}$ & $\mathrm{SD}$ & $\mathrm{F}$ & $p$ & $t$ & df & $p$ \\
\hline \multicolumn{10}{|c|}{ Verbal Memory } \\
\hline RAVLT (immediate recall) & 37.51 & 4.85 & 23.81 & 7.24 & 1.721 & 0.20 & 6.67 & 33.00 & 0.000 \\
\hline $\begin{array}{l}R A V L T \\
\text { (delayed recall) }\end{array}$ & 6.10 & 1.40 & 3.31 & 3.36 & 9.273 & 0.00 & 3.10 & 19.33 & 0.006 \\
\hline RAVLT correct recognitions & 14.79 & 0.42 & 10.50 & 3.98 & 30.544 & 0.00 & 4.29 & 15.28 & 0.001 \\
\hline RAVLT false recognitions & 0.58 & 0.77 & 2.19 & 2.86 & 11.846 & 0.00 & 2.19 & 16.83 & 0.043 \\
\hline \multicolumn{10}{|c|}{ Episodic Memory } \\
\hline $\begin{array}{l}B S R \\
\text { (immediate recall) }\end{array}$ & 4.88 & 0.63 & 2.96 & 1.77 & 6.421 & 0.02 & 4.13 & 18.23 & 0.001 \\
\hline $\begin{array}{l}\text { BSR } \\
\text { (delayed recall) }\end{array}$ & 4.83 & 0.67 & 2.51 & 2.36 & 30.615 & 0.00 & 3.80 & 17.05 & 0.001 \\
\hline \multicolumn{10}{|c|}{ Verbal working memory } \\
\hline DS & 4.99 & 0.58 & 4.63 & 0.50 & 1.209 & 0.28 & 1.96 & 33.00 & 0.059 \\
\hline \multicolumn{10}{|c|}{ Visual memory } \\
\hline $\begin{array}{l}\text { Rey-Osterrieth's figure } \\
\text { (immediate recall) }\end{array}$ & 15.06 & 7.11 & 7.53 & 6.48 & 0.043 & 0.84 & 3.25 & 33.00 & 0.003 \\
\hline $\begin{array}{l}\text { Rey-Osterrieth's figure } \\
\text { (delayed recall) }\end{array}$ & 15.08 & 7.42 & 7.41 & 6.09 & 0.118 & 0.73 & 3.30 & 33.00 & 0.002 \\
\hline \multicolumn{10}{|c|}{ Selective Attention } \\
\hline VS & 46.95 & 5.15 & 44.31 & 8.31 & 3.932 & 0.06 & 1.15 & 33.00 & 0.260 \\
\hline \multicolumn{10}{|c|}{ Attentional shift } \\
\hline TMT & 44.16 & 26.09 & 137.38 & 74.60 & 15.01 & 0.00 & 4.76 & 18.09 & 0.000 \\
\hline \multicolumn{10}{|c|}{ Language } \\
\hline$V P F$ & 30.95 & 8.17 & 24.25 & 8.15 & 0.081 & 0.78 & 2.42 & 33.00 & 0.021 \\
\hline VSF & 35.00 & 5.64 & 28.81 & 10.00 & 8.058 & 0.01 & 2.20 & 22.74 & 0.038 \\
\hline$B N T$ & 29.53 & 0.61 & 26.75 & 2.24 & 25.510 & 0.00 & 4.82 & 16.89 & 0.000 \\
\hline \multicolumn{10}{|c|}{ Visuo-constructional skills } \\
\hline Clock Test & 1.00 & 0.00 & 2.81 & 1.28 & 35.561 & 0.00 & 5.68 & 15.00 & 0.000 \\
\hline $\begin{array}{l}\text { Rey-Osterrieth's figure } \\
\text { (copy) }\end{array}$ & 33.97 & 2.68 & 23.25 & 11.59 & 24.597 & 0.00 & 3.62 & 16.35 & 0.002 \\
\hline \multicolumn{10}{|c|}{ Executive functions } \\
\hline$F A B$ & 18.00 & 0.00 & 14.25 & 3.19 & 55.298 & 0.00 & 4.70 & 15.00 & 0.000 \\
\hline \multicolumn{10}{|c|}{ Logic and abstract reasoning skills } \\
\hline$R C P M$ & 27.04 & 3.51 & 22.57 & 4.21 & 1.652 & 0.21 & 3.38 & 32.00 & 0.002 \\
\hline
\end{tabular}

MCImd, multi-domain mild cognitive impairment; C, control; RAVLT, Rey auditory verbal learning test; BSR, Babcock Story Recall; DS, Digit span; VS, Visual search; TMT, Trail making test; VPF, Verbal phonemic fluency test; VSF, Verbal semantic fluency test; BNT, Boston Naming Test; FAB, Frontal Assessment Battery; RCPM, Raven's Colored Progressive Matrices.

using the CBT [29]. This test consists of 9 wooden blocks $(4.5 \times 4.5 \mathrm{~cm})$ fixed on a board $(30 \times 25 \mathrm{~cm})$ in a scattered array. On the experimenter's side, the blocks were numbered for easy identification. The examiner tapped a sequence of blocks at the rate of one block every 2 seconds, after which the participant had to reproduce the same sequence in the same order. In the working memory task, different sequences of increasing length (starting from 2-block sequences) were presented until the subject failed to reproduce two out of three trials of a given length. A span score corresponding to the longest sequence the subject was able to correctly reproduce was calculated. In the learning task, the examiner showed the same sequence of eight spatial locations that the participant had to learn and recall at each presentation (maximum number of trials: 18). The learning criterion corresponded to three consecutive correct reproductions of the sequence with no demonstration by the experimenter. When the subject was able to correctly repeat the sequence three times in a row, the examiner stopped the presentation. Scoring was computed as follows: the examiner counted the number of the blocks correctly tapped for each of the 18 trials. The learning score corresponded to the sum of blocks correctly tapped in each trial (maximum score: 144). Thus, unlike the span score, which corresponds to the longest sequence that participants can 
retain and reproduce, the learning score corresponds to the number of positions of a given sequence participants learn. After an interval of five minutes, the examiner asked the subject to reproduce the sequence learned previously. The number of blocks tapped correctly corresponds to the visuo-spatial delayed recall (VSDR; maximum score: 8).

\section{Topographical memory: Navigational vista space}

The WalCT [27, 28] was used to assess topographical working memory (TWM) and topographical immediate (TL) and delayed recall (TDR) learning in navigational vista space. This test, which is a larger version of the CBT $(3 \times 2.5 \mathrm{~m}$; scale $1: 10$ of the CBT), consists of 9 black flat squares placed on the floor in the same positions as the standard CBT. In the TWM task, the examiner illustrated, as was done in the CBT, sequences of increasing length (starting from a 2-square sequence) by walking on the floor and stopping on each square for two seconds. The subject was then required to repeat the same sequence as the examiner, moving from the same starting point and walking and stopping on the same squares. The span score was the longest sequence participants were able to correctly reproduce in three trials out of five. In the learning task, the examiner showed, as was done in the VSL, an 8-square spatial sequence that participants had to learn and recall (maximum number of trials: 18). The learning criterion was the same as that used in the CBT, i.e., three correct trials in a row with no demonstration by the examiner. The number of squares correctly reproduced was calculated for each trial. The learning score corresponded to the sum of squares tapped correctly (maximum score 144). One point was attributed to each square reproduced correctly until the criterion was fulfilled; this score was then added to the score corresponding to correct performance in the remaining trials (up to the 18th).

The TDR of the learned sequence was assessed after five minutes; the number of squares tapped correctly corresponded to the topographical delayed recall (maximum score: 8 ).

\section{Navigational skills in real world environmental space}

\section{Route navigation}

To test navigational skills in the real world, we asked participants to learn an indoor/outdoor path within the grounds of the "Umberto I" University
Hospital in Rome (Fig. 1A). This task was administered in daylight on sunny days to ensure that both the indoor and outdoor areas were well lit. The path included 3 straight stretches, 3 left turns, and 5 right turns on the ground floor. The examiner showed the subject the path, telling him/her to pay attention because he/she would have to follow the path described at the end of the demonstration. At the end of the description, the participants performed the landmark recognition task (see below) and were then required to reproduce the route without the experimenter's guidance. The experimenter always followed the subject, remaining a few steps away so as to avoid providing any help and to allow the participants to navigate on their own. If they made an error, they were allowed to wander for 3 minutes before being led back to the last correct turn and being asked to try again from that point. No help or suggestions were provided while they wandered for the 3 minutes. The proportion of correct turns was then computed for each participant.

\section{Landmark recognition}

After following the experimenter along the route, participants were asked to decide whether the pictures they were shown were those of the landmarks $(n=8)$ they had encountered along the path (e.g., a door) or whether they were distractors (e.g., a door that was similar to the landmark, $n=8$ ) (Fig. 1B). The sum of correct responses yielded the correct score for each participant (maximum score: 16).

\section{Statistical analyses}

\section{Group statistics}

Statistical analyses were performed by using SPSS 25. Performances on working memory (VSWM and TWM), learning (VSL and TL), and delayed recall (VSDR and TDR) within reaching and navigational vista spaces were entered as dependent variables in three different mixed factorial ANOVAs, with Group (C versus $\mathrm{MCI}$ ) as the between-subject factor and Space (Reaching space versus Navigational Vista space) as the within-subject factor. Two-sample $t$ tests were used to compare the performances of MCI and $\mathrm{C}$ in the route navigation and landmark recognition tasks. Levene's test was adopted to assess equality of variances; where significance departure from equality of variance was detected, we did not assume equality of variance. The main corpus of analyses was performed on the whole sample of MCI (i.e., including both MCImd and MCIsd) and C. To 

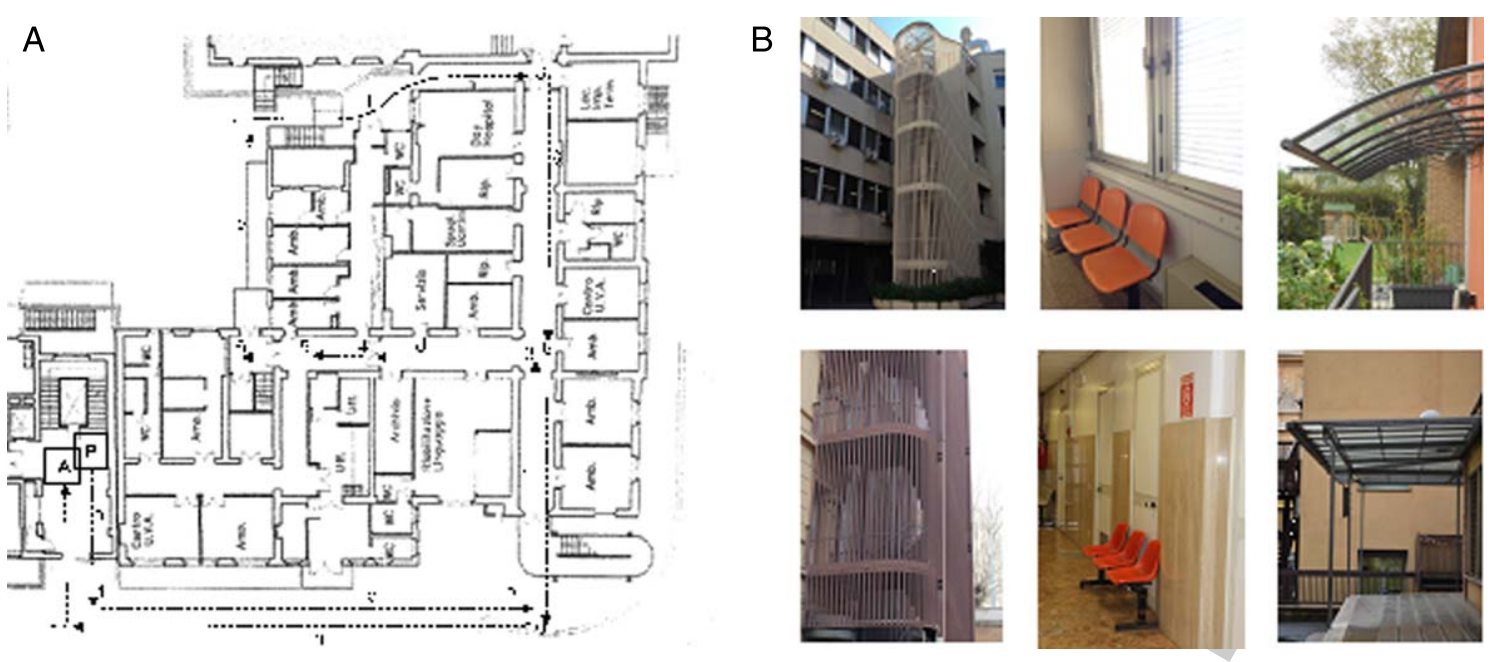

Fig. 1. Assessment of navigational skills in ecological environment. A) In/out-door path within the hospital used to assess route learning skill. Turns are numbered (1-11) whereas stretches are labelled as letters. B) Examples of landmarks encountered along the path (on the bottom of the panel) and fillers (on the top of the panel).

investigate any results due to differences in the neuropsychological profiles of MCIsd and MCImd, as a check we ran the same corpus of analyses on MCImd as a group and MCIsd as single cases (see "Single case analysis" paragraph below). We also tested whether MCImd and C differed in any of the neuropsychological tests by performing two-sample $t$-tests using the same procedure as that adopted for testing differences in navigational tasks in the ecological environment. The full results are shown in Table 2.

\section{Single case analysis}

For each MCIsd patient and memory domain (i.e., working memory, learning, and delayed recall), we also ran single-case analyses using DISSOCSBAYES.EXE to test for Bayesian criteria for dissociations [47] between the reaching and navigational vista spaces, namely between VSWM and TWM, VSL and TL, and VSDR and TDR. Moreover, the performances of MCIsd in navigational tasks (i.e., route navigation and landmark recognition tasks) were compared with those of $\mathrm{C}$ using a one-tailed $t$-test modified procedure implemented in Singlims.exe, which computes point estimate and confidence limits to test for the abnormality of a test score $[48,49]$. MCIsd scores in the neuropsychological tests are shown in Table 3.

\section{Correlation analysis}

Pearson correlation coefficients were also computed for the whole MCI sample to assess the relationship between memory deficits detected by standard neuropsychological tests (i.e., immediate and delayed recall of Rey auditory verbal learning test, Babcock Story Recall, Rey-Osterrieth's figure; digit span) and memory deficits detected by visuospatial and topographical tests (i.e., VSWM, VSL, VSDR, TWM, TL, TDR, route navigation).

\section{RESULTS}

\section{Group results}

As regards working memory span, we found a main effect of Group $\left(\mathrm{F}_{1,36}=21.35 ; p<0.001\right.$; $\eta_{\mathrm{p}}{ }^{2}=0.37$; observed power $\left.=0.99\right)$, with MCI performing worse than $\mathrm{C}$, and Space $\left(\mathrm{F}_{1,36}=67.89\right.$; $p<0.001 ; \eta_{\mathrm{p}}^{2}=0.65$; observed power $\left.=1.00\right)$, with better performances on VSWM than on TWM (Fig. 2A). We did not observe any Group-by-Space interaction $\left(\mathrm{F}_{1,36}=2.72 ; \quad p=0.108 ; \quad \eta_{\mathrm{p}}{ }^{2}=0.07\right.$; observed power $=0.36$ ). As regards learning, we found a main effect of Group $\left(\mathrm{F}_{1,36}=19.56\right.$; $p<0.001 ; \quad \eta_{\mathrm{p}}^{2}=0.352 ; \quad$ observed power $=0.99$ ), with MCI performing worse than $\mathrm{C}$, and Space $\left(\mathrm{F}_{1,36}=24.68 ; \quad p<0.001 ; \quad \eta_{\mathrm{p}}{ }^{2}=0.41 ; \quad\right.$ observed power $=1.00$ ), with better performances on VSL than on TL. The Group-by-Space interaction was also significant $\left(\mathrm{F}_{1,36}=6.15 ; p=0.018 ; \eta_{\mathrm{p}}{ }^{2}=0.15\right.$; observed power $=0.67$ ): post-hoc pairwise comparisons showed that TL skills were worse than VSL skills in MCI $(p<0.001$, adjusted for multiple comparisons using Bonferroni's correction); by contrast, C performed similarly on VSL and TL 
Table 3

MCIsd scores in neuropsychological and navigational tests. Pathological scores are marked in bold

\begin{tabular}{|c|c|c|c|}
\hline Test/Patient & MCIsd1 & MCIsd2 & MCsdI3 \\
\hline \multicolumn{4}{|c|}{ Verbal Memory } \\
\hline RAVLT (immediate recall) & 25.00 & 19.00 & 37.00 \\
\hline RAVLT (delayed recall) & 1.00 & 0.00 & 4.00 \\
\hline RAVLT correct recognitions & 8.00 & 6.00 & 14.00 \\
\hline RAVLT false recognitions & 1.00 & 0.00 & 6.00 \\
\hline \multicolumn{4}{|c|}{ Episodic Memory } \\
\hline BSR (immediate recall) & 3.60 & 0.00 & 5.80 \\
\hline BSR (delayed recall) & 3.20 & 2.00 & 0.00 \\
\hline \multicolumn{4}{|c|}{ Verbal working Memory } \\
\hline DS & 8.00 & 5.00 & 5.00 \\
\hline \multicolumn{4}{|c|}{ Visual Memory } \\
\hline Rey-Osterrieth's figure (immediate recall) & 6.50 & 1.00 & 20.00 \\
\hline Rey-Osterrieth's figure (delayed recall) & 6.00 & 0.00 & 22.00 \\
\hline \multicolumn{4}{|c|}{ Selective Attention } \\
\hline VS & 48.00 & 57.00 & 44.00 \\
\hline \multicolumn{4}{|c|}{ Attentional shift } \\
\hline TMT & 38.00 & 198.00 & 21.00 \\
\hline \multicolumn{4}{|c|}{ Language } \\
\hline VPF & 24.00 & 20.00 & 44.00 \\
\hline VSF & 39.00 & 25.00 & 49.00 \\
\hline BNT & 30.00 & 26.00 & 29.00 \\
\hline \multicolumn{4}{|c|}{ Visuo-constructional skills } \\
\hline Clock Test & 1.00 & 2.00 & 1.00 \\
\hline Rey-Osterrieth's figure (copy) & 36.00 & 36.00 & 33.10 \\
\hline \multicolumn{4}{|c|}{ Executive functions } \\
\hline FAB & 18.00 & 13.00 & 18.00 \\
\hline \multicolumn{4}{|c|}{ Logic and abstract reasoning skills } \\
\hline RCPM & 33.00 & 29.00 & 29.00 \\
\hline \multicolumn{4}{|c|}{ Navigational tasks } \\
\hline TWM & 3.00 & 2.00 & 4.00 \\
\hline VSWM & 5.00 & 4.00 & 5.00 \\
\hline TL & 68.00* & $45.00^{*}$ & 127.00 \\
\hline VSL & $106.00^{*}$ & $80.00^{*}$ & 140.00 \\
\hline TDR & $1.00^{\#}$ & 5.00 & 8.00 \\
\hline VSDR & 3.00 & $4.00^{\#}$ & 6.00 \\
\hline Route navigation & 0.80 & NA & 0.78 \\
\hline Landmark recognition & 14.00 & 11.00 & 13.00 \\
\hline
\end{tabular}

MCIsd, single-domain mild cognitive impairment; C, control; RAVLT, Rey auditory verbal learning test; BSR, Babcock Story Recall; DS, Digit span; VS, Visual search; TMT, Trail making test; VPF, Verbal phonemic fluency test; VSF, Verbal semantic fluency test; BNT, Boston Naming Test; FAB, Frontal Assessment Battery; RCPM, Raven's Colored Progressive Matrices. *Performances in these tasks are dissociated. \# performances in these tasks are not dissociated.

( $p=0.087$, adjusted for multiple comparisons using Bonferroni's correction) (Fig. 2B). This result suggests that the two skills are at least in part dissociated in MCI. Finally, ANOVA on delayed recall revealed a main effect of Group $\left(\mathrm{F}_{1,36}=17.15\right.$; $p<0.001 ; \eta_{\mathrm{p}}{ }^{2}=0.32$; observed power $=0.98$ ), with MCI performing worse than $\mathrm{C}$ (Fig. 2C). No other significant effect was detected (Space: $\mathrm{F}_{1,36}=0.27$; $p=0.607 ; \eta_{\mathrm{p}}{ }^{2}=0.01$; observed power $=0.08$; Spaceby-Group: $\quad \mathrm{F}_{1,36}=2.428 ; \quad p=0.128 ; \quad \eta_{\mathrm{p}}{ }^{2}=0.06$; observed power $=0.33$ ).
The set of analyses in the MCImd subgroup yielded the same pattern of results. We detected a main effect of Group $\left(\mathrm{F}_{1,33}=32.90 ; p<0.001 ; \eta_{\mathrm{p}}{ }^{2}=0.50\right.$; observed power $=1.00)$ and Space $\left(\mathrm{F}_{1,33}=58.37\right.$; $p<0.001 ; \quad \eta_{\mathrm{p}}^{2}=0.64 ;$ observed power $\left.=1.00\right)$ on working memory: MCImd patients performed significantly worse than $\mathrm{C}$ while performances on VSWM were better than those on TWM (Fig. 2D). The Group-by-Space interaction was not significant $\left(\mathrm{F}_{1,33}=2.42 ; p=0.129 ; \eta_{\mathrm{p}}{ }^{2}=0.07 ;\right.$ observed power $=0.327$ ). We detected a main effect of Group 
A

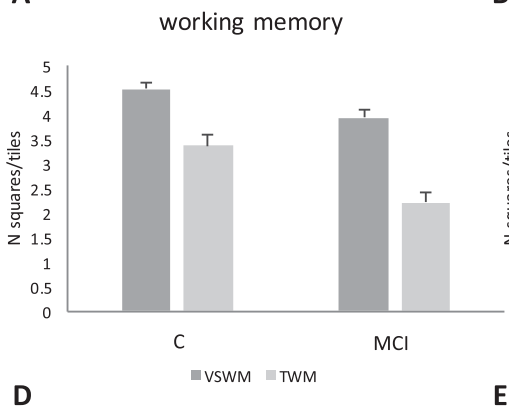

working memory

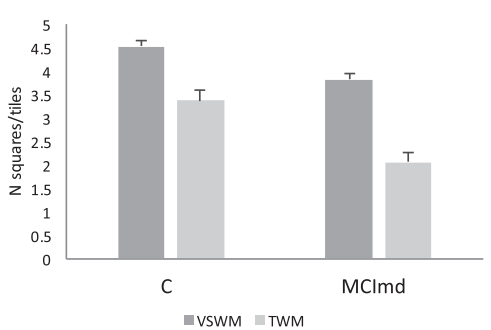

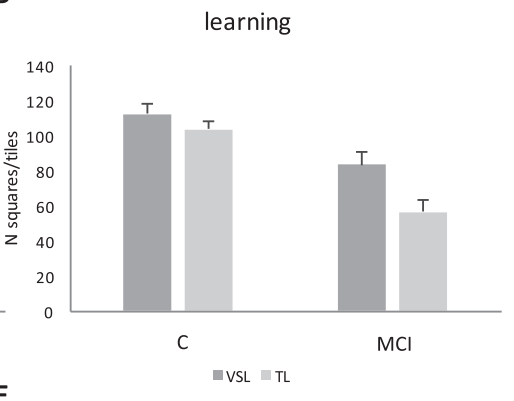

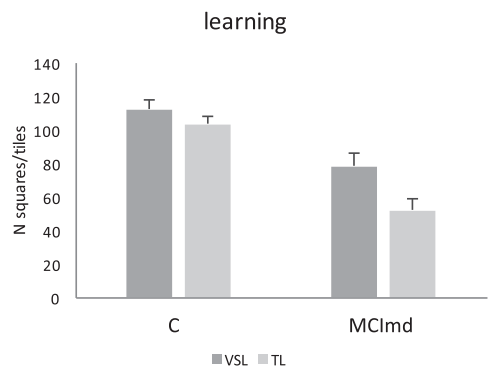

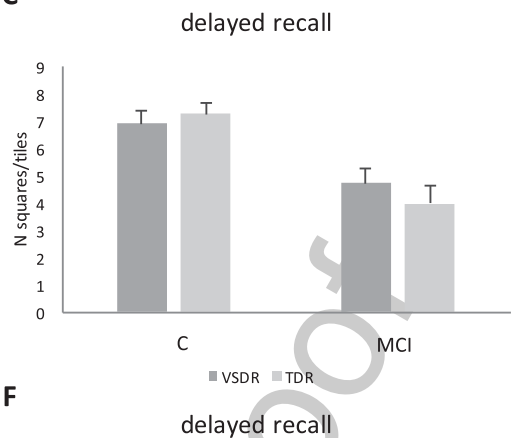

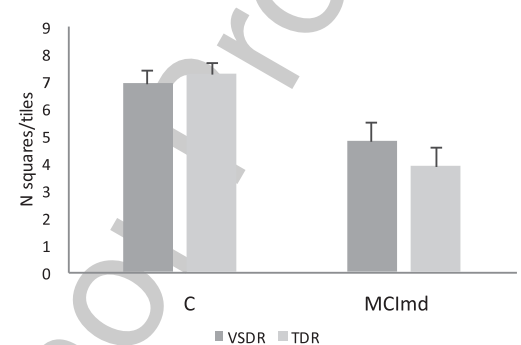

Fig. 2. Bars show the mean group performances and standard error in each memory component. Results of the general analysis on $\mathrm{C}$ and MCI groups are shown in panels A-C; the results of comparisons between the MCImd subgroup and C are shown in panels D-F. C, healthy controls; MCI, amnestic mild cognitive impairment; MCImd, multi-domain MCI.

and Space on learning skill (Group: $\mathrm{F}_{1,33}=25.32$; $p<0.001 ; \eta_{\mathrm{p}}{ }^{2}=0.43$; observed power $=1.00$; Space: $\mathrm{F}_{1,33}=20.67 ; \quad p<0.001 ; \quad \eta_{\mathrm{p}}{ }^{2}=0.39 ; \quad$ observed power $=0.99$ ), with MCImd performing worse than $\mathrm{C}$, and performances on VSL being better than those on TL (Fig. 2E). The Group-by-Space interaction was again significant $\left(\mathrm{F}_{1,33}=5.03 ; p=0.032\right.$; $\eta_{\mathrm{p}}{ }^{2}=0.13$; observed power $=0.59$ ): post-hoc pairwise comparisons showed that TL skills were worse than VSL skills in MCImd $(p<0.001$, adjusted for multiple comparisons using Bonferroni's correction); C instead performed similarly on VSL and TL ( $p=0.098$, adjusted for multiple comparisons using Bonferroni's correction) (Fig. 2E). Finally, ANOVA on delayed recall revealed a main effect of Group $\left(\mathrm{F}_{1,33}=16.00 ; \quad p<0.001 ; \quad \eta_{\mathrm{p}}{ }^{2}=0.33 ; \quad\right.$ observed power $=0.97$ ), with MCImd performing worse than $\mathrm{C}$ (Fig. 2F). No other significant effect was detected (Space: $\mathrm{F}_{1,33}=0.58 ; p=0.451 ; \eta_{\mathrm{p}}{ }^{2}=0.02$; observed power $=0.12$; Group-by-Space interaction: $\mathrm{F}_{1,33}=3.07 ; p=0.089 ; \eta_{\mathrm{p}}{ }^{2}=0.09 ;$ observed power $=0.40$ ).

As regards navigational tasks in the ecological environment, $\mathrm{MCI}(\mathrm{M}=0.83 ; \mathrm{SD}=0.16)$ performed worse than $\mathrm{C}(\mathrm{M}=0.95 ; \mathrm{SD}=0.07)$ in the route navigation task $\left(\mathrm{t}_{23.92}=2.93 ; p=0.007\right.$; variance is not assumed as equal due to significant Levene's test, $\mathrm{F}=9.48 ; p=0.004)$. By contrast, no difference was detected between MCI $(\mathrm{M}=10.26$; $\mathrm{SD}=2.00)$ and $\mathrm{C}(\mathrm{M}=10.95 ; \mathrm{SD}=1.81)$ in the landmark recognition task $\left(\mathrm{t}_{36}=1.11 ; p=0.276\right)$. The same pattern of results was observed in MCImd (route navigation: $\mathrm{t}_{19.97}=2.54 ; p=0.019$; variance is not assumed as equal due to significant Levene's test, $\mathrm{F}=13.08 ; p=0.001$; landmark recognition: $\left.\mathrm{t}_{33}=1.88 ; p=0.070\right)$ : MCImd $(\mathrm{M}=0.84 ; \mathrm{SD}=0.17)$ performed worse than $\mathrm{C}$ in the route navigation task though not in the landmark recognition task (MCImd: $\mathrm{M}=9.81, \mathrm{SD}=1.76$ ).

\section{Single case results}

A detailed description of the demographic characteristics of MCIsd is provided in Table 1. MCIsd1 performed similarly to $\mathrm{C}$ (13 females) in working memory in both VSWM $(t=1.39, \mathrm{df}=12$, $p($ one-tailed $)=0.095)$ and TWM $(\mathrm{t}=-0.40, \mathrm{df}=12$, $p($ one-tailed $)=0.350)$; however, she fulfilled the criteria for a dissociation in learning $(p=0.014)$ by performing within the normal range in VSL $(\mathrm{t}=-0.37, \mathrm{df}=12, \mathrm{p}($ one-tailed $)=0.358)$ though not in $\mathrm{TL}(\mathrm{t}=-2,665, \mathrm{df}=12, p($ one-tailed $)=0.010)$. As her delayed recall was impaired in both VDR $(\mathrm{t}=-1.85, \mathrm{df}=12, p($ one-tailed $)=0044)$ and TDR $(\mathrm{t}=-4.60, \mathrm{df}=12, p($ one-tailed $)<0.001)$, she did not fulfil the criteria for dissociation in this ability $(p=0.080)$. 
MCIsd2 performed similarly to C (13 females) in working memory in both VSWM $(\mathrm{t}=-0.62$, $\mathrm{df}=12, p($ one-tailed $)=0.274)$ and TWM $(\mathrm{t}=-1.25$, $\mathrm{df}=12, p($ one-tailed $)=0.118)$. MCIsd2 also fulfilled the criteria for a dissociation in learning $(p=0.010)$ by performing within the normal range in VSL $(\mathrm{t}=-1.362, \mathrm{df}=12, p($ one-tailed $)=0.099)$ though not in $\mathrm{TL}(\mathrm{t}=-4.15, \mathrm{df}=12, \mathrm{p}$ (one-tailed $)<0.001)$. As regards delayed recall, she performed within the normal range in VSDR $(\mathrm{t}=-1.40, \mathrm{df}=12, p$ (onetailed $)=0.095)$ but worse than $\mathrm{C}$ in TDR $(\mathrm{t}=-1.82$, $\mathrm{df}=12, p($ one-tailed $)=0.047)$ without, however, fulfilling the criteria for either a strong or classical dissociation $(p=0.741)$.

MCIsd3 performed similarly to $\mathrm{C}$ (6 males) in working memory (VSWM: $\mathrm{t}=0.00, \mathrm{df}=5$, $p($ one-tailed $)=0.500$; TWM: $\mathrm{t}=1.22, \mathrm{df}=5, p($ onetailed $)=0.138)$, learning $(\mathrm{VSL}: \mathrm{t}=1.09, \mathrm{df}=5$, $p($ one-tailed $)=0.162 ;$ TL: $\mathrm{t}=1.09, \mathrm{df}=5, p($ onetailed $)=0.164)$ and delayed recall (VSDR: $\mathrm{t}=-0.31$, $\mathrm{df}=5, p($ one-tailed $)=0.383 ;$ TDR: $\mathrm{t}=0.14, \mathrm{df}=5$, $p($ one-tailed $)=0.446)$.

As regards the navigational tasks, MCIsd1 performed worse than $\mathrm{C}$ (13 females) in the route navigation task $(\mathrm{t}=2.03 ; \mathrm{df}=12 ; p($ one-tailed $)=0.033)$ though not in the landmark recognition task $(\mathrm{t}=1.69$; $\mathrm{df}=12 ; p($ one-tailed $)=0.058)$. MCIsd 2 performed as well as $\mathrm{C}$ (13 females) in the landmark recognition task $(\mathrm{t}=0.04 ; \mathrm{df}=12 ; p($ one-tailed $)=0.483)$; data on route learning were not available because the patient did not complete the protocol. MCIsd3 performed slightly worse than $\mathrm{C}$ ( 6 males) in the route navigation $\operatorname{task}(\mathrm{t}=1.88 ; \mathrm{df}=5 ; p($ one-tailed $)=0.059)$ and similarly to $\mathrm{C}$ in the landmark recognition task $(\mathrm{t}=0.88$; $\mathrm{df}=5 ; p($ one-tailed $)=0.209)$.

\section{Correlational analysis}

Pearson's correlation coefficients are reported in Table 4. In MCI, TWM and TL were both significantly positively correlated with immediate Babcock Story Recall whereas VSWM and VSL were not. VSWM was instead positively correlated with performances in the digit span test. Moreover, TWM, TL, TDR and VSDR were all positively correlated with performances on immediate recall in Rey-Osterrieth's figure. TWM also positively correlated with performances on delayed recall in Rey-Osterrieth's figure. Performances on TDR were negatively correlated with performances in the digit span test.

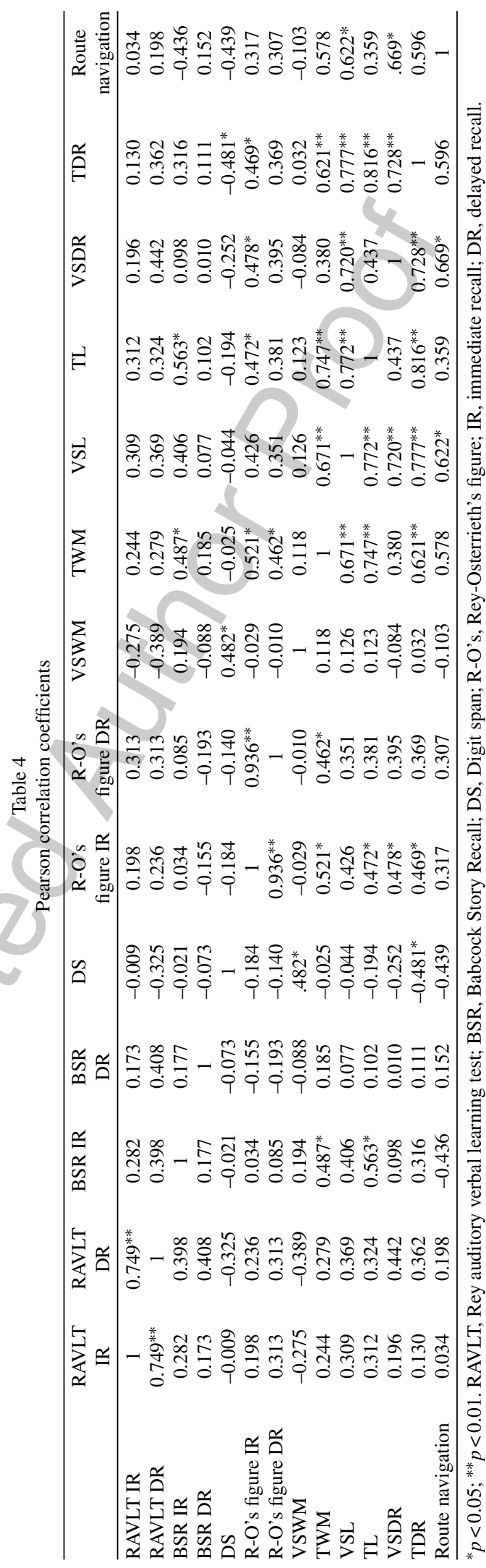




\section{DISCUSSION}

Increasing life expectancy and the accompanying rise in the prevalence of age-related cognitive disorders makes the identification of accurate markers of pathological aging of paramount importance. Spatial navigation is a promising tool for the early detection of pathological aging. Here we provide evidence of the usefulness of navigational tasks in the neuropsychological assessment of MCI patients. We focused on dimensions known to be involved in pathological aging, such as topographical memory (assessed by means of the WalCT) and real world navigation (assessed by using route navigation and landmark recognition tasks) $[10,30,31]$ and which we also expected to be impaired in MCI.

As regards topographical memory, ANOVA on performances in working memory, learning and delayed recall tasks revealed a main effect of Space, with performances proving to be worse in the WalCT than in CBT. This indicates that memory of positions in navigational vista space is more demanding than visuo-spatial memory within reaching space, a finding that is likely due to the fact that the former requires a change in perspective, i.e., egocentric perspective taking, during the reproduction of the path sequence, which is not instead required during the reproduction of the sequence in reaching space. Thus, memory of positions in navigational vista space may be more sensitive to mild deficits in preclinical populations. The fact that MCI performed worse than $\mathrm{C}$ in all the memory domains points to a general deficit in recalling spatial position within both reaching and navigational vista spaces. However, the Group-by-Space interaction we detected in the learning scores suggests that topographical learning is more severely compromised in MCI. Indeed, with the exception of a main effect of Group in the topographical and visuo-spatial memory tasks, we found that MCI patients, unlike C, performed worse in TL than in VSL. Accordingly, the dissociation we detected among MCIsd between TL and VSL, with a selective deficit in TL and the sparing of VSL, is in line with the group results and points to an early and selective deficit in TL in MCIsd. If developmental trajectories are considered, this finding is worthy of note insofar as it shows that memory in navigational space develops later and declines earlier than that in reaching space $[50,51]$. A possible explanation for this difference between reaching and navigational vista space is related to the loss of environmental autonomy that older people may experience in everyday life. Indeed, this problem manifests itself even in healthy individuals with developmental topographical disorientation, who become fully aware of their difficulty when they move autonomously in the environment or when they face situations in which they have to be active in spatial orientation $[52,53]$. However, it is noteworthy that healthy older adults perform similarly in learning positions in both reaching and navigational vista space [54], even if their span in both spaces is shorter than that of young adults. This absence of differentiation between the two types of space in normal aging suggests that it is possible to detect the loss of topographical memory only in early pathological aging, probably owing to a reversal trend in the developmental processes underlying topographical memory acquisition during childhood [50, 51].

With regard to real world navigation in environmental space, we found that MCI patients performed worse than $\mathrm{C}$ in the route navigation task despite being spared in the landmark recognition task. This result suggests that topographical disorientation, which is frequently reported in $\mathrm{AD}$ and $\mathrm{MCI}$, is not due to a deficit in landmark processing and/or landmark agnosia, but mainly involves memory for routes and positions within navigational environment. This hypothesis is confirmed by the single case analyses on MCIsd patients and supports the idea that a deficit in spatial navigation skills is already present in the prodromal stages of $\mathrm{AD}$. One possible explanation for this finding is an impairment in the egocentric frame of reference. Indeed, the integrity of egocentric frames of reference is widely acknowledged to be crucial to the correct functioning of daily behavior in space [55-57]. When individuals orient themselves through egocentric frames of reference, they use the body as anchor points to collect spatial information of the surrounding space; by contrast, allocentric frames of reference define the position of a target in relation to an environmental landmark, which acts as an anchor point $[58,59]$. Besides providing evidence of an early deficit in the allocentric frame of reference in $\mathrm{AD}$ and $\mathrm{MCI}$ patients, Bianchini and colleagues [10] showed that the egocentric frame of reference is also misused in early-stage AD. They found that TWM in patients with early-stage AD was impaired whereas VSWM was spared. The results of the present study extend their findings by highlighting the presence of a deficit not only in TWM but also in learning and recall in an 8-step path, with a dissociation between reaching and navigational vista spaces being observed in the prodromal stages of $\mathrm{AD}$, e.g., in MCIsd. 
The analyses on MCImd (as a group) and MCIsd (treated as single cases) confirm the results of the main group comparisons (MCI versus C). MCImd patients were impaired in all the tasks (with the exception of landmark recognition) and performed worse when learning spatial positions within navigational vista space than within reaching space. Interestingly, the single case analyses on three patients with a selective memory deficit, i.e., MCIsd, expand upon this result by revealing a dissociation between learning spatial positions within navigational vista space and learning positions within reaching space, with the former being selectively impaired in two patients who thus satisfied the criteria for dissociation. Taken together, these results deserve considerations based on both clinical and theoretical perspectives.

From a clinical point of view, finding that $\mathrm{TL}$ is more severely compromised than VSL in MCImd and selectively compromised in MCIsd strongly supports the use of TL tests in clinical practice, especially in the prodromal stages of $\mathrm{AD}$ (i.e., amnestic single domain MCI, MCIsd). On the one hand, it will provide a more comprehensive picture of memory deficits in MCI and AD. On the other hand, it will allow the topographical disorientation of which patients complain to be quantified, which is a highly relevant issue owing to the impact of topographical disorientation in the activities of daily living among healthy elderly subjects and MCI patients. Indeed, elderly people who are aware of these difficulties are widely known to adopt strategies to avoid unknown environments [60], thereby reducing the opportunities they may have to get to know new environments and to experience active navigation. Since topographical disorientation has a considerable impact on the lives of patients and their caregivers, it is very important to study its characteristics and to evaluate which populations these disorders affect so as to be able to make a diagnosis as early as possible. Topographical disorientation may even contribute to functional decline and accelerate the conversion from the MCI status to dementia. Memory tests within navigational vista space may help to gain a better understanding of navigational deficits in the elderly and their progression to MCI. Notwithstanding the large number of studies pointing to specific deficits in environmental navigation in MCI patients, real-world navigation is not included in the routine neuropsychological assessment. The neuropsychological assessment instead mainly consists of visuo-spatial memory tasks within reaching space, which are dissociated from topographical learning in MCIsd and are more severely compromised in MCImd. Furthermore, in keeping with other preliminary results on the predictive power of spatial navigation in $\mathrm{AD}[61,32]$ indicating that topographical learning is compromised in the early phase of MCIsd while visuo-spatial learning is spared, the results of the present study suggest that TL may be a promising tool both for making an early diagnosis and for monitoring disease progression in preclinical and early AD.

From a theoretical point of view, it is interesting to note that learning spatial positions within navigational vista space and reaching space are dissociated in MCIsd and impaired in varying ways in MCImd. This finding is in line with the segregated neural networks highlighted by Nemmi et al. [62] for learning positions within near and far space by means of fMRI in healthy participants: they found that within the large occipito-parieto-frontal network underlying learning of positions within reaching and navigational vista spaces, the right lingual gyrus, calcarine sulcus and dorsolateral prefrontal cortex were specifically associated with learning in navigational vista space, whereas the left inferior temporal gyrus, lingual and fusiform gyrus and middle occipital gyrus were associated with learning sequences in reaching space. Taken together, these results suggest that a number of cognitive dimensions warrant investigation in MCI and $\mathrm{AD}$, two diseases characterized by patterns of atrophy [63] that mainly affect brain areas involving spatial navigation and topographical memory. This dissociation is also consistent with a series of studies in the field of cognitive psychology that have revealed differences in memory for positions within reaching and navigational vista spaces, thereby demonstrating that the CBT and WalCT assess two different types of visuo-spatial memory [64-66].

It is also worth noting that, from a theoretical point of view, performances involving topographical working memory and learning, though not those involving visuospatial working memory and learning, were significantly correlated with performances involving immediate recall of Babcock Story. Although this finding appears to suggest that the topographical memory deficit we detected in MCI is mainly due to a general memory deficit, we did not find topographical working memory and learning correlated with other memory scores, such as Rey Auditory Verbal learning. Topographical memory instead selectively correlated with recalling structured verbal material, such as a story. This result 
fits in well with the hypothesis made by Buzsaki and Moser [67], according to which the phylogenetic roots of planning and memory mechanisms lie within mechanisms of spatial navigation in the physical world. Upon reviewing the literature, these authors suggested that hippocampus-dependent memories evolved from mechanisms introduced to compute relationships between environmental positions (e.g., firing patterns allowing for spatiotemporal ordering and the chunking of landmarks and positions, such as those of the place and grid cells). The correlation between story recall and topographical memory we observed in this study is in keeping with their hypothesis: the loss of firing patterns that underlie coding positions and spatial relations within the environment as a result of hippocampal atrophy in MCI may be directly linked to the deficit in episodic memory observed in such patients. Although this interpretation is tentative and deserves further investigations into the causal relationship between topographical and memory deficits in MCI, the present results strongly support the idea that MCI is a neuropsychological model that may be used to disentangle this relationship.

We did not explore the effect of gender in the present study because the purpose of our study was to investigate differences between normal and pathological aging as opposed to gender-related differences. Thus, we developed our navigational tasks in an ecological environment (i.e., route navigation and landmark recognition) according to the methods used in previous studies, which did not report any gender differences [68]. Furthermore, as gender-related differences are not expected for learning scores in the WalCT [28], in the present study we did not expect to detect gender-related differences in tasks designed to investigate spatial navigation within ecological environment and topographical learning. Gender-related differences are instead to be expected for span scores in the WalCT [28]. However, the number of participants we enrolled in this study did not allow for gender-based statistical comparisons. Further studies are needed to investigate this issue and understand whether and, if so, how gender interacts with aging in determining performances involving topographical working memory.

\section{Conclusions}

In conclusion, our results show that topographical memory and route based-navigation may represent markers of pathological aging. By using clinical tools aimed at investigating these aspects, it is possible to detect navigational disorders that may be early predictors of cognitive decline. Further studies on patients with MCI are needed to identify which MCI patients are most likely to convert to AD and which navigational components are damaged in those patients who do convert to AD. Spatial navigation is a multifaceted ability that may be used to identify impaired critical processes in patients with MCI and consequently to shed light on neuropsychological markers of cognitive decline.

\section{DISCLOSURE STATEMENT}

Authors' disclosures available online (https:// www.j-alz.com/manuscript-disclosures/18-0890r1).

\section{REFERENCES}

[1] Klein DA, Steinberg M, Galik E, Steele C, Sheppard JM, Warren A, Rosenblatt A, Lyketsos CG (1999) Wandering behaviour in community-residing persons with dementia. Int J Geriatr Psychiatry 14, 272-279.

[2] Kirasic KC (1991) Spatial cognition and behavior in young and elderly adults: Implications for learning new environments. Psychol Aging 6, 10-18.

[3] Wilkniss SM, Jones MG, Korol DL, Gold PE, Manning CA (1997) Age- related differences in an ecologically based study of route learning. Psychol Aging 12, 372-375.

[4] Iaria G, Palermo L, Committeri G, Barton JJ (2009) Age differences in the formation and use of cognitive maps. Behav Brain Res 196, 187-191.

[5] Moffat SD (2009) Aging and spatial navigation: What do we know and where do we go? Neuropsychol Rev 19, 478-489.

[6] DeIpolyi AR, Rankin KP, Mucke L, Miller BL, GornoTempini ML (2007) Spatial cognition and the human navigation network in AD and MCI. Neurology 79, 986-997.

[7] Monacelli AM, Cushman LA, Kavcic V, Duffy CJ (2003) Spatial disorientation in Alzheimer's disease: The remembrance of things passed. Neurology 61, 1491-1497.

[8] Bird CM, Chan D, Hartley T, Pijnenburg YA, Rossor MN, Burgess N (2010) Topographical short-term memory differentiates. Alzheimer's disease from frontotemporal lobar degeneration. Hippocampus 20, 1154-1169.

[9] Wolbers T, Hegarty M (2010) What determines our navigational abilities? Trends Cogn Sci 14, 138-146.

[10] Bianchini F, Di Vita A, Palermo L, Piccardi L, Blundo C, Guariglia C (2014) A selective egocentric topographical working memory deficit in the early stages of alzheimer's disease: A preliminary study. Am J Alzheimers Dis Other Demen 29, 749-754.

[11] Janzen G, Jansen C (2010) A neural wayfinding mechanism adjusts for ambiguous landmark information. Neuroimage 52, 364-370.

[12] Nemmi F, Boccia M, Guariglia C (2017) Does aging affect the formation of new topographical memories? Evidence from an extensive spatial training. Neuropsychol Dev Cogn B Aging Neuropsychol Cogn 24, 29-44.

[13] Boccia M, Silveri MC, Sabatini U, Guariglia C, Nemmi F (2016) Neural underpinnings of the decline of topographical memory in mild cognitive impairment. Am J Alzheimers Dis Other Demen 31, 618-630. 
[14] Iachini T, Iavarone A, Senese VP, Ruotolo F, Ruggiero G (2009) Visuospatial memory in healthy elderly, AD and MCI: A review. Curr Aging Sci 2, 43-59.

[15] Gazova I, Laczó J, Rubinova E, Mokrisova I, Hyncicova E, Andel R, Vyhnalek M, Sheardova K, Coulson EJ, Hort J (2013) Spatial navigation in young versus older adults. Front Aging Neurosci 5, 94.

[16] Lithfous S, Dufour A, Després O (2013) Spatial navigation in normal aging and the prodromal stage of Alzheimer's disease: Insights from imaging and behavioral studies. Ageing Res Rev 12, 201-213.

[17] Serino S, Cipresso P, Morganti F, Riva G (2014) The role of egocentric and allocentric abilities in Alzheimer's disease: A systematic review. Ageing Res Rev 16, 32-44.

[18] Guariglia CC, Nitrini R (2009) Topographical disorientation in Alzheimer's disease. Arq Neuropsiquiatr 67, 967-972.

[19] McShane R, Gedling K, Keene J, Fairburn C, Jacoby R, Hope T (1998) Getting lost in dementia: A longitudinal study of a behavioral symptom. Int Psychogeriatr 10, 253260.

[20] Pai MC, Jacobs WJ (2004) Topographical disorientation in community-residing patients with Alzheimer's disease. Int $J$ Geriatr Psychiatry 19, 250-255.

[21] Weniger G, Ruhleder M, Lange C, Wolf S, Irle E (2011) Egocentric and allocentric memory as assessed by virtual reality in individuals with amnestic mild cognitive impairment. Neuropsychologia 49, 518-527.

[22] Uc EY, Rizzo M, Anderson SW, Shi Q, Dawson JD (2004) Driver route-following and safety errors in early Alzheimer disease. Neurology 63, 832-837.

[23] Rosenbaum RS, Gao F, Richards B, Black SE, Moscovitch M (2005) "Where to?" Remote memory for spatial relations and landmark identity in former taxi drivers with Alzheimer's disease and encephalitis. J Cogn Neurosci 17, 446-462.

[24] Peter J, Sandkamp R, Minkova L, Schumacher LV, Kaller CP, Sbdulkadir A, Klöppel S (2018) Real-world navigation in amnestic mild cognitive impairment: The relation to visuospatial memory and volume of hippocampal subregions. Neuropsychologia 109, 86-94.

[25] Boccia M, Nemmi F, Guariglia C (2014) Neuropsychology of environmental navigation in humans: Review and meta-analysis of FMRI studies in healthy participants. Neuropsychol Rev 24, 236-251.

[26] Wolbers T \& Wiener JM (2014) Challenges for identifying the neural mechanisms that support spatial navigation: The impact of spatial scale. Front Hum Neurosci 8, 571.

[27] Piccardi L, Iaria G, Ricci M, Bianchini F, Zompanti L, Guariglia C (2008). Walking in the Corsi test: Which type of memory do you need? Neurosci Lett 432, 127-131.

[28] Piccardi L, Bianchini F, Argento O, De Nigris A, Maialetti A, Palermo L, Guariglia C (2013) The Walking Corsi Test (WalCT): Standardization of the topographical memory test in an Italian population. Neurol Sci 34, 971-978.

[29] Corsi PM (1972) Human memory and the medial temporal region of the brain. Unpublished doctoral dissertation. McGill University, Montreal, Quebec.

[30] Allison SL, Fagan AM, Morris JC, Head D (2016) Spatial navigation in preclinical Alzheimer's disease. J Alzheimers Dis 52, 77-90.

[31] Verghese J, Lipton R, Ayers E (2017) Spatial navigation and risk of cognitive impairment: A prospective cohort study. Alzheimers Dement 13, 985-992.

[32] Rusconi ML, Suardi A, Zanetti M, Rozzini L (2015) Spatial navigation in elderly healthy subjects, amnestic and non amnestic MCI patients. J Neurol Sci 359, 430-437.
[33] Albert MS, DeKosky ST, Dickson D, Dubois B, Feldman, HH, Fox NC, Gamst A, Holtzman DM, Jagust WJ, Petersen RC, Snyder PJ, Carrillo MC, Thies B, Phelps CH (2011) The diagnosis of mild cognitive impairment due to Alzheimer's disease: Recommendations from the National Institute on Aging-Alzheimer's Association workgroups on diagnostic guidelines for Alzheimer's disease. Alzheimers Dement 7, 270-279.

[34] Folstein MF, Folstein SE, McHugh PR (1975) “Mini-mental state". A practical method for grading the cognitive state of patients for the clinician. J Psychiatr Res 12, 189-198.

[35] Rosen WG, Mohs RC, Davis KL (1984) A new rating scale for Alzheimer's disease. Am J Psychiatry 141, 1356-1364.

[36] Carlesimo GA, Buccione I, Fadda L, Graceffa A, Mauri M, Lorusso S, Bevilacqua G, Caltagirone C (2002) Standardizzazione di due test di memoria per uso clinico: Breve Racconto e Figura di Rey. Nuova Riv Neurol 12, 1-3.

[37] Orsini A, Grossi D, Capitani E, Laiacona M, Papagno C, Vallar G (1987) Verbal and spatial immediate memory span: Normative data from 1355 adults and 1112 children. Ital $J$ Neurol Sci 8, 539-548.

[38] Spinnler H, Tognoni G (1987) Standardizzazione e taratura italiana di test neuropsicologici. Ital J Neurol Sci Suppl 8, $1-120$.

[39] Giovagnoli AR, Del Pesce M, Mascheroni S, Simoncelli M, Laiacona M, Capitani E (1996) Trail Making Test: Normative values from 287 normal adults controls. Ital J Neurol Sci 17, 305-309.

[40] Novelli G, Papagno C, Capitani E, Laiacona N, Vallar G, Cappa SF (1986) Tre test clinici di ricerca e produzione lessicale: Taratura su soggetti normali. Arch Psicol Neurol Psichiatr 47, 477-506.

[41] Van Gorp WG, Satz P, Kiersch ME, Henry R (1986) Normative data on the Boston Naming Test for a group of normal older adults. J Clin Exp Neuropsychol 8, 702-705.

[42] Appollonio I, Leone M, Isella V, Piamarta F, Consoli T, Villa ML, Forapani E, Russo A, Nichelli P (2005) The Frontal Assessment Battery (FAB): Normative values in an Italian population sample. Neurol Sci 26, 108-116.

[43] Basso A, Capitani E, Laiacona M (1987) Raven's coloured progressive matrices: Normative values on 305 adult normal controls. Funct Neurol 2, 189-194.

[44] Raven JC (1938) Standard Progressive Matrices: Sets A, B, C, D and EHK. London: Lewis.

[45] Shulman KI, Pushkar Gold D, Cohen CA, Zucchero CA (1993) Clock-drawing and dementia in the community: A longitudinal study. Int J Geriatr Psychiatry 8, 487-496.

[46] Lawton MP, Brody EM (1969) Assessment of older people: Self-maintaining and instrumental activities of daily living. Gerontologist 9, 179-186.

[47] Crawford JR, Garthwaite PH (2007) Comparison of a single case to a control or normative sample in neuropsychology: Development of a Bayesian approach. Cogn Neuropsychol 24, 343-372.

[48] Crawford JR, Garthwaite PH (2002) Investigation of the single case in neuropsychology: Confidence limits on the abnormality of test scores and test score differences. $\mathrm{Neu}$ ropsychologia 40, 1196-1208.

[49] Crawford JR, Howell DC (1998) Comparing an individual's test score against norms derived from small samples. Clin Neuropsychol 12, 482-486.

[50] Piccardi L, Palermo L, Leonzi M, Risetti M, Zompanti L, D'Amico S, Guariglia C (2014) The Walking Corsi Test (WalCT): Normative study on topographical working memory in a 4 to 11-year old children sample. Clin Neuropsychol 28, 84-96. 
[51] Piccardi L, Leonzi M, D’Amico S, Guariglia C (2014) Developmental of navigational working memory: Evidence from 6- to 10- year-old children. Br J Dev Psychol 32, 205-217.

[52] Bianchini F, Incoccia C, Palermo L, Piccardi L, Zompanti L, Sabatini U, Peran P, Guariglia C (2010) Developmental topographical disorientation in a healthy subject. Neuropsychologia 48, 1563-1573.

[53] Piccardi L, De Luca M, Di Vita A, Palermo L, Tanzilli A, Dacquino C, Pizzamiglio MR (2017) Evidence of taxonomy for developmental topographical disorientation: Developmental landmark agnosia case 1. Appl Neuropsychol Child 1, 1-12.

[54] Piccardi L, Iaria G, Bianchini F, Zompanti L, Guariglia C (2011) Dissociated deficits of visuo-spatial memory in near space and navigational space: Evidence from brain-damaged patients and healthy older participants. $\mathrm{Neu}$ ropsychol Dev Cogn B Aging Neuropsychol Cogn 18, 362-384.

[55] Aguirre GK, D’Esposito M (1999) Topographical disorientation: A synthesis and taxonomy. Brain 122, 1613-1628.

[56] Burgess N (2006) Spatial memory: How egocentric and allocentric combine. Trends Cogn Sci 10, 551-557.

[57] Galati G, Pelle G, Berthoz A, Committeri G (2010) Multiple reference frames used by the human brain for spatial perception and memory. Exp Brain Res 206, 109-120.

[58] Kosslyn SM (1994) Image and brain: The resolution of the imagery debate. MIT Press, Cambridge, MA.

[59] McNamara TP (2003) How are the locations of objects in the environment represented in memory? In Spatial cognition III, routes and navigation, human memory and learning, spatial representation and spatial learning, Freksa C, Brauer W, Habel C, Wender K, eds. Springer-Verlag, Berlin, Germany, pp. 174-191.
[60] Burns PC (1999) Navigation and the mobility of older drivers. J Gerontol B Psychol Sci Soc Sci 54, S49-55.

[61] Bellassen V, Igloi K, de Souza LC, Dubois B, Rondi-Reig L (2012) Temporal order memory assessed during spatiotemporal navigation as a behavioral cognitive marker for differential Alzheimer's disease diagnosis. J Neurosci 32, 1942-1952.

[62] Nemmi F, Boccia M, Piccardi L, Galati G, Guariglia C (2013) Segregation of neural circuits involved in spatial learning in reaching and navigational space. Neuropsychologia 51, 1561-1570.

[63] Boccia M, Acierno M, Piccardi L (2015) Neuroanatomy of Alzheimer's disease and late-life depression: A coordinatebased meta-analysis of MRI studies. J Alzheimers Dis 46, 963-970.

[64] Palmiero M, Piccardi L (2017) The role of emotional landmarks on topographical memory. Front Psychol 8, 763.

[65] Piccardi L, Berthoz A, Baulac M, Denos M, Dupont S, Samson S, Guariglia C (2010) Different spatial memory systems are involved in small-and large-scale environments: Evidence from patients with temporal lobe epilepsy. Exp Brain Res 206, 171-177.

[66] Piccardi L, Nori R, Boccia M, Barbetti S, Verde P, Guariglia C, Ferlazzo F (2015) A dedicated system for topographical working memory: Evidence from domain-specific interference tests. Exp Brain Res 233, 2489-2495.

[67] Buzsaki G, Moser EI (2013) Memory, navigation and theta rhythm in the hippocampal-entorhinal system. Nat Neurosci 16, 130-138.

[68] Boccia M, Vecchione F, Piccardi L, Guariglia C (2017) Effect of cognitive style on learning and retrieval of navigational environments. Front Pharmacol 8, 496. 